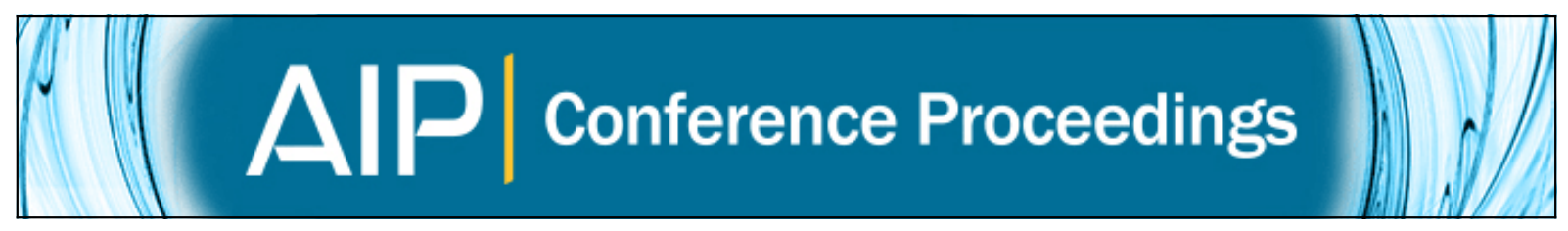

\title{
Excitation of wakefields in a relativistically hot plasma created by dying non-linear plasma wakefields
}

A. A. Sahai, T. C. Katsouleas, S. Gessner, M. Hogan, C. Joshi, and W. B. Mori

Citation: AIP Conference Proceedings 1507, 618 (2012); doi: 10.1063/1.4773768

View online: http://dx.doi.org/10.1063/1.4773768

View Table of Contents: http://scitation.aip.org/content/aip/proceeding/aipcp/1507?ver=pdfcov

Published by the AIP Publishing

\section{Articles you may be interested in}

Combined effects of gas pressure and exciting frequency on electron energy distribution functions in hydrogen capacitively coupled plasmas

Phys. Plasmas 20, 023501 (2013); 10.1063/1.4789611

Relativistic effects in the interaction of high intensity ultra-short laser pulse with collisional underdense plasma Phys. Plasmas 18, 093108 (2011); 10.1063/1.3633529

Self-consistent nonlinear transverse waves in relativistic plasmas

Phys. Plasmas 13, 062303 (2006); 10.1063/1.2207123

Nonlinear viscosity and its role in drift-Alfvén modes

Phys. Plasmas 12, 122509 (2005); 10.1063/1.2151169

Relativistic electron beam acceleration by nonlinear Landau damping of electrostatic waves in a magnetized plasma

Phys. Plasmas 11, 5634 (2004); 10.1063/1.1812537 


\title{
Excitation Of Wakefields In A Relativistically Hot Plasma Created By Dying Non-linear Plasma Wakefields
}

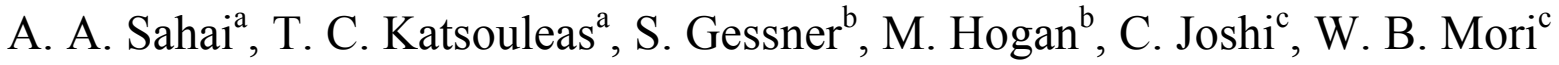 \\ ${ }^{a}$ Electrical and Computer Engineering, Duke University, Durham, NC 27708, USA \\ ${ }^{b}$ SLAC National Accelerator Laboratory, Menlo Park, CA 90309, USA \\ ${ }^{c}$ University of California Los Angeles, Los Angeles, CA 90095, USA
}

\begin{abstract}
We study the various physical processes and their timescales involved in the excitation of wakefields in relativistically hot plasma. This has relevance to the design of a high repetition-rate plasma wakefield collider in which the plasma has not had time to cool between bunches in addition to understanding the physics of cosmic jets in relativistically hot astrophysical plasmas. When the plasma is relativistically hot (plasma temperature near $\mathrm{m}_{\mathrm{e}} \mathrm{c}^{2}$ ), the thermal pressure competes with the restoring force of ion space charge and can reduce or even eliminate the accelerating field of a wake. We will investigate explicitly the case where the hot plasma is created by a preceding Wakefield drive bunch 10's of picoseconds to many nanoseconds ahead of the next drive bunch. The relativistically hot plasma is created when the excess energy (not coupled to the driven $\mathrm{e}^{-}$bunch) in the wake driven by the drive $\mathrm{e}^{-}$bunch is eventually converted into thermal energy on 10's of picosecond timescale. We will investigate the thermalization and diffusion processes of this non-equilibrium plasma on longer time scales, including the effects of ambi-polar diffusion of ions driven by hot electron expansion, possible Columbic explosion of ions producing higher ionization states and ionization of surrounding neutral atoms via collisions with hot electrons. Preliminary results of the transverse and longitudinal wakefields at different timescales of separation between a first and second bunch are presented and a possible experiment to study this topic at the FACET facility is described.
\end{abstract}

Keywords: Relativistically Hot Plasma, Plasma Wakefield Collider, High repetition rate, Dying Plasma Wakefield, FACET.

\section{ELECTRON BEAM INTERACTION WITH RELATIVISTICALLY HOT PLASMA}

High-energy high intensity electron beam interacting with appropriate density plasma can excite non-linear "blowout" Wakefields. A trailing or witness bunch loaded in right phase of the Wakefields, where transverse focusing and longitudinal acceleration fields are both at their peak, can maximally absorb the energy and accelerate high quality beam in the drive bunch generated Wakefields.

The trailing witness bunch or the bunch being accelerated cannot however absorb all the energy coupled by the drive bunch into the plasma Wakefields. The maximum energy transfer efficiency is of the order $30-40 \%$. Hence, even in the cases where the witness bunch accelerates with optimal characteristics, there is excess energy left over in the plasma. This excess energy leads to different processes during the dying stages of the plasma wakefields. The ions that are left in the ion column created by ponderomotively-ejected electrons tend to diffuse out away from the axis. This leads to the possibility of existence of a hollow plasma channel. At later timescales, the plasma tends to thermalize with energy of electrons of the order of their initial ponderomotive force balance.

Facility for ACcelerator Experiments and Test (FACET) at Stanford Linear Accelerator Center (SLAC) National Laboratory is a unique facility for advancing accelerator technology as well as the frontiers of science. The proposed experiments of studying the dying stages of the Plasma Wakefields accomplish both of these goals. It will explore the feasibility of running a plasma wakefield accelerator at high repetition rate, while at the same time explore new beam physics of relativistic beams interacting with extremely hot and possibly turbulent plasmas typically found only in astrophysical settings.

Advanced Accelerator Concepts

AIP Conf. Proc. 1507, 618-622 (2012); doi: 10.1063/1.4773768

(C) 2012 American Institute of Physics 978-0-7354-1125-8/\$30.00 
One of the main motivations for building FACET was that it would allow the development and testing of many aspects of a single stage of a future multi-stage Plasma Linear Collider (PLC) at the energy frontier. One of the first self-consistent designs for a high luminosity PLC is shown in Figure 1 with parameters given in Table 1.

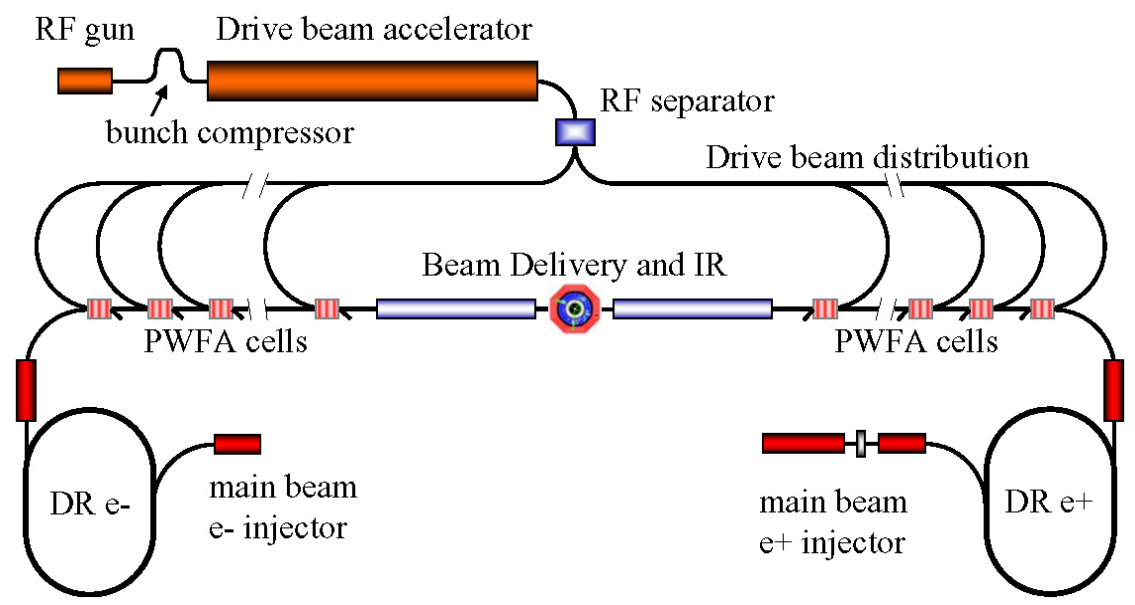

Figure 1. Self consistent design of PLC

The design is for collision energy of $1 \mathrm{TeV}$ in the center of mass with $20 \mathrm{e}^{+}$and $\mathrm{e}^{-}$plasma stages, each of approximately the energy of the FACET plasma Wakefield experiments (E-200). To achieve luminosity above $10^{34}$ $\mathrm{cm}^{-2} \mathrm{~s}^{-1}$ requires each plasma stage to repeat every 4 nanoseconds. In such a short time, the plasma will be strongly affected by the thermalization of the wake from the previous pulses. Open questions include what will be the temperature and density profile of such plasma? Will the unabsorbed wake energy from the previous pulse(s) heat the plasma to relativistic temperatures (i.e., $\mathrm{k}_{\mathrm{B}} \mathrm{T}_{\mathrm{e}}$ of the order or greater than $0.5 \mathrm{MeV}$, thermal velocities near the speed of light)? Can wakes be excited and used for acceleration in such plasma and how will they differ from the cold plasma wakes studied thus far? How long must one wait for the plasma to cool, diffuse, recombine, etc. before it can be re-used effectively? This experiment will be the first to explore high repetition rate plasma acceleration and answer these questions.

Moreover, the interaction of relativistic beams with relativistic plasmas is of interest for understanding the physics of astrophysical jets (Figure 2). In recent years, new observational facilities for astrophysics have revealed that such jets are ubiquitous in nature, emerging from objects ranging from pulsars to supernovae and galactic nuclei. Their interaction with surrounding hot plasma gasses exhibits self-focusing, hosing, radiation generation and other phenomena that can be studied in this proposed experiment.

Table 1. PLC design parameters

\begin{tabular}{|l|l|}
\hline Main beam: bunch population, bunches per train, rate & $1 \times 10^{10}, 125,100 \mathrm{~Hz}$ \\
\hline Total power of two main beams & $20 \mathrm{MW}$ \\
\hline Drive beam: energy, peak current and active pulse length & $25 \mathrm{GeV}, 2.3 \mathrm{~A}, 10 \mu \mathrm{s}$ \\
\hline Average power of the drive beam & $58 \mathrm{MW}$ \\
\hline Plasma density, accelerating gradient and plasma cell length & $1 \times 10^{17} \mathrm{~cm}^{-3}, 25 \mathrm{GV} / \mathrm{m}, 1 \mathrm{~m}$ \\
\hline Power transfer efficiency drive beam=>plasma =>main beam & $35 \%$ \\
\hline Efficiency: Wall plug=>RF=>drive beam & $50 \% \times 90 \%=45 \%$ \\
\hline Overall efficiency and wall plug power for acceleration & $15.7 \%, 127 \mathrm{MW}$ \\
\hline Site power estimate (with 40MW for other subsystems) & $170 \mathrm{MW}$ \\
\hline Main beam emittances, $\mathrm{x}, \mathrm{y}$ & $2,0.05 \mathrm{~mm}-\mathrm{mrad}$ \\
\hline Main beam sizes at Interaction Point, $\mathrm{x}, \mathrm{y}, \mathrm{z}$ & $0.14,0.0032,10 \mu \mathrm{m}$ \\
\hline Luminosity & $3.5 \times 10^{34} \mathrm{~cm}^{-2} \mathrm{~s}^{-1}$ \\
\hline Luminosity in $1 \%$ of energy & $1.3 \times 10^{34} \mathrm{~cm}^{-2} \mathrm{~s}^{-1}$ \\
\hline
\end{tabular}




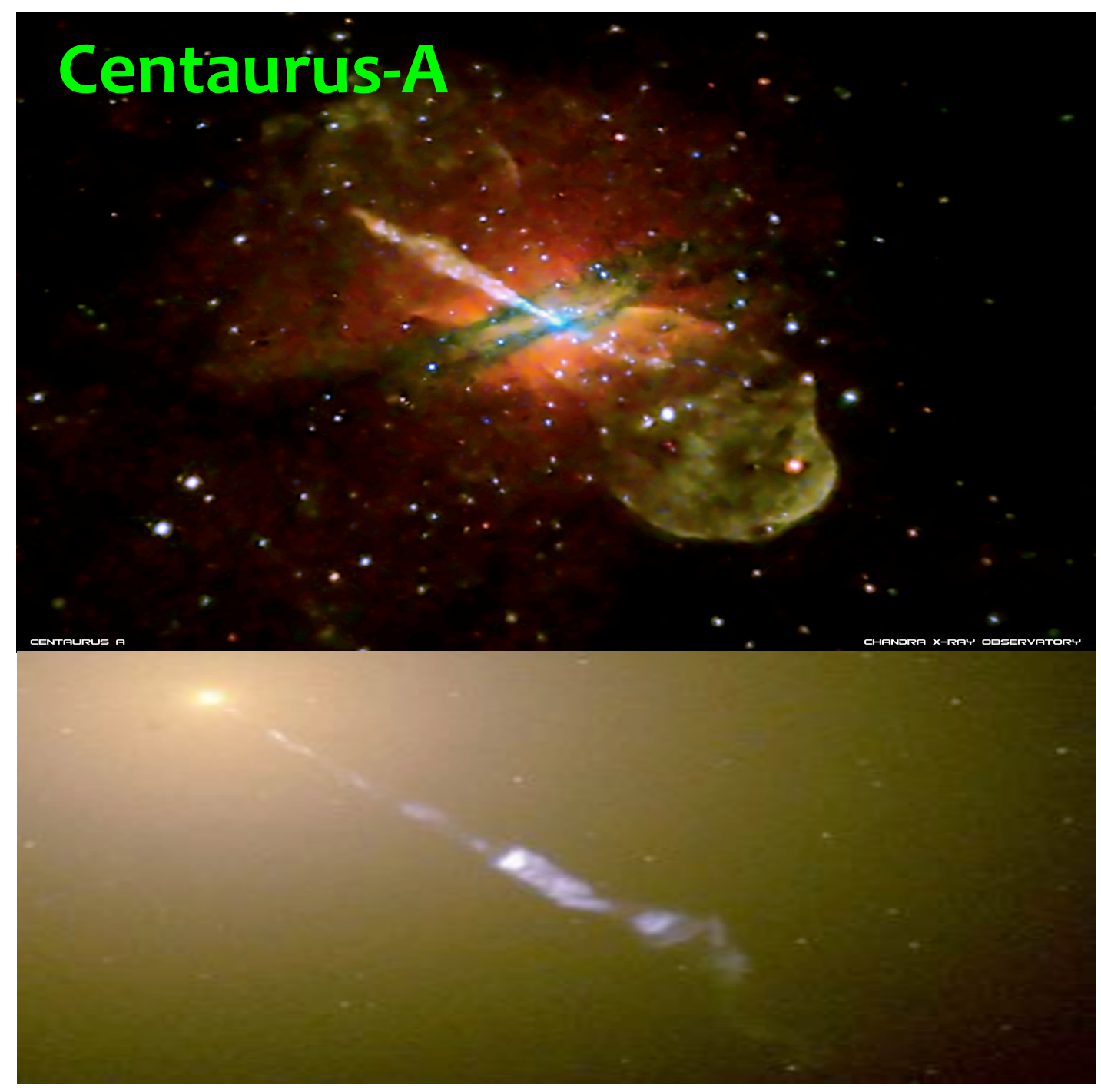

Figure 2. Astrophysical Jets from gravitationally compressing hot plasmas in Galaxies

To illustrate the physics that may occur in a high rep-rate plasma Wakefield stage, we perform 2-D particle-in-cell simulations (with cylindrical symmetry assumptions) with the well-benchmarked code OSIRIS. Figure 3 shows real space iso-contours of longitudinal plasma longitudinal Wakefield $E_{x}$ in a cold plasma for typical E-200 experimental parameters as well as corresponding wakes in plasmas of increasing temperature from 0 to a few $\mathrm{MeV}$. At relativistic temperatures, the mass of plasma electrons increases, lowering the plasma frequency $\left(\omega_{\mathrm{pe}}=\sqrt{ }\left\{4 \pi \mathrm{n}_{0} \mathrm{e}^{2} /\right.\right.$ $\left.\gamma_{\mathrm{e}} \mathrm{m}_{\mathrm{e}}\right\}$ ) where $\gamma_{\mathrm{e}}$ is the Lorentz factor of the hot electrons) and correspondingly increasing the plasma wavelength $\left(\lambda_{\mathrm{p}}=2 \pi \mathrm{c} / \omega_{\mathrm{pe}}\right)$ and wake radius (proportional to the skin depth $\left.\mathrm{c} / \omega_{\mathrm{pe}}\right)$. The Wakefield amplitude is also reduced in accordance with the lower $\omega_{\mathrm{pe}}$, and the "spike" in electric field (at the back of the bubble) characteristic of cold plasma wakes of large amplitude is lower because thermal pressure of the relativistic electrons prevents the density from compressing to the same degree. 

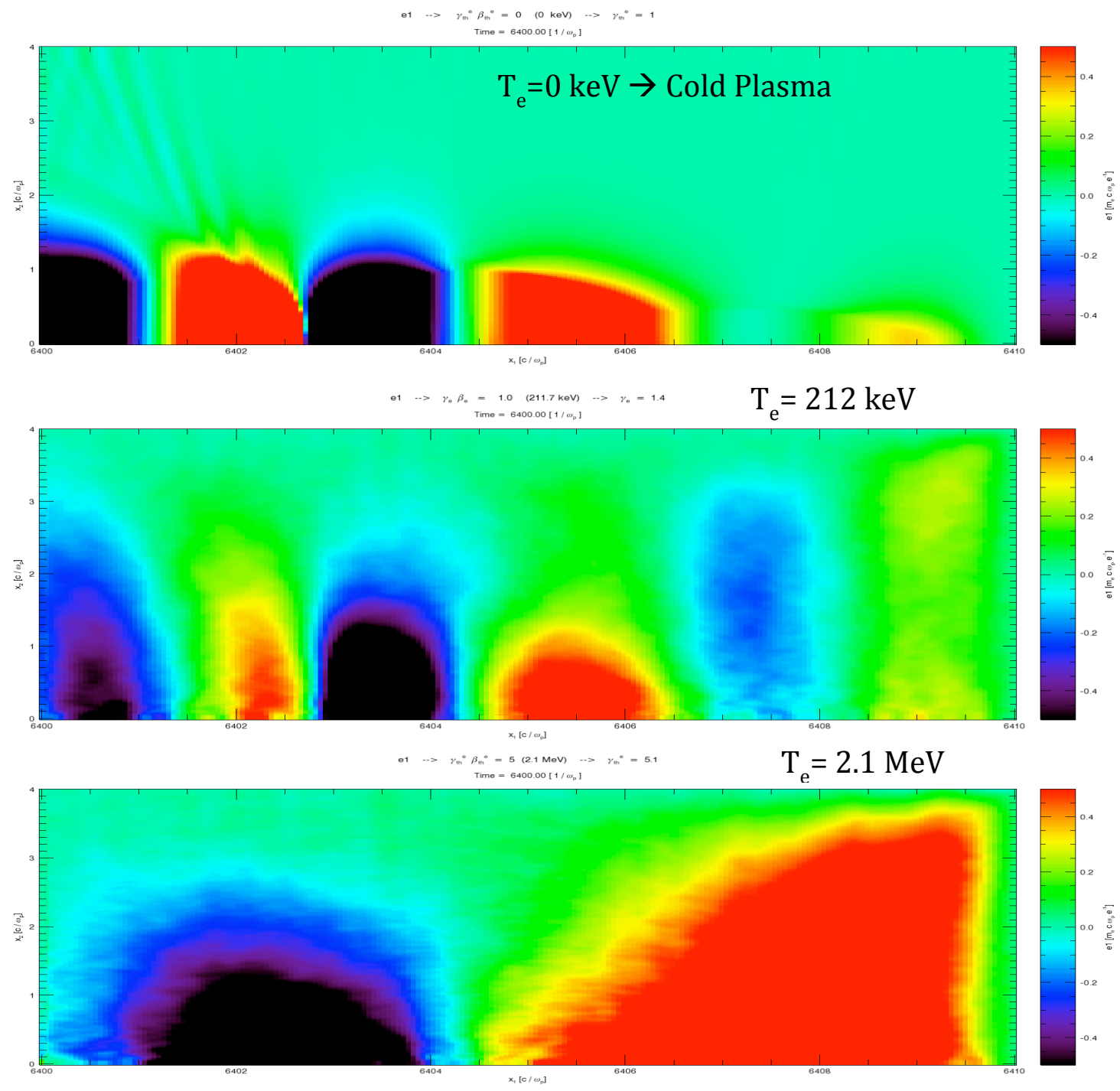

Figure 3. Longitudinal Wakefields $\mathrm{E}_{\mathrm{x}}$ in Hot plasma (electron temperature in eV) in a real-space Cross-section (iso-contours on one side of the axis)

The actual temperature that the plasma could reach in a high-rep accelerator depends on a number of factors including how well the wake energy is absorbed through efficient beam loading. As an upper limit, one can estimate that when the wake energy thermalizes due to plasma instabilities and mode couplings, the kinetic energy of the oscillating electrons in the wake (as well as some of the field energy) will become random thermal energy. Roughly the quiver velocity of electrons in the wake approaches $\rightarrow \mathrm{c} \times \mathrm{E}_{\mathrm{x}}[\mathrm{V} / \mathrm{cm}] / \sqrt{ }\left(\mathrm{n}_{0}\left[\mathrm{~cm}^{-3}\right]\right)$. For FACET the latter ratio is of order $1\left(\mathrm{E}_{\mathrm{x}} \sim 10 \mathrm{GV} / \mathrm{m} ; \mathrm{n}_{0} \sim 10^{17} \mathrm{~cm}^{-3}\right)$, so we may expect temperatures of several hundred $\mathrm{keV}$ to $\mathrm{MeV}$ after the wake thermalizes.

The thermalization of a large amplitude plasma wake is complex and little studied. Previous PIC simulations have shown that the wake decoheres on a timescale of 10's of plasma periods or a few times $10^{-3} / \sqrt{ }\left(\mathrm{n}_{0}\right) \sim 10$ picosecond scale. The resulting hot plasma electrons will expand outward driving so called ambi-polar diffusion of the ions at a speed typically near the sound speed $\mathrm{c}_{\mathrm{s}} \sim \sqrt{ }\left(\mathrm{T}_{\mathrm{e}} / \mathrm{M}_{\mathrm{i}}\right)$, where $\mathrm{T}_{\mathrm{e}}$ is the electron temperature and $\mathrm{M}_{\mathrm{i}}$ is the ion mass. For $\mathrm{MeV}$ electrons and Rubidium ions, this is a few $\mathrm{mm} / \mathrm{ns}$. This will lead to a typically parabolic channel of lowered plasma density on axis on a nano-second timescale. However, as the plasma expands it cools, slowing the process and eventually allowing recombination to occur (microsecond timescale). 


\section{ACKNOWLEDGMENTS}

Work supported by the National Science Foundation under NSF-PHY-0936278, NSF-PHY-0904039 and NSFPHY-0936266, the US Department of Energy under DEFC02-07ER41500 and DE-FG02-92ER40727, and the DOE Fusion Science Center through a University of Rochester Subcontract No.415025-G.

\section{REFERENCES}

1. A. Seryi, M. Hogan, S. Pei, T. Raubenheimer, P. Tenenbaum, T. Katsouleas, C. K. Huang, C. Joshi, W. Mori, P. Muggli, A Concept Of Plasma Wake Field Acceleration Linear Collider (PWFA-LC). Proceedings of PAC09, Vancouver, BC, Canada. WE6PFP081

2. M. Hogan, T O Raubenheimer, A Seryi, P Muggli, T Katsouleas, C Huang, W Lu, W An, K A Marsh, W B Mori, C E Clayton and C Joshi, Plasma wakefield acceleration experiments at FACET, 2010 New J. Phys. 12055030

3. Kaw, P. K., Dawson, J.M., Relativistic nonlinear propagation of laser beams in cold over dense plasmas. Phys. Fluids 13, 472, (1970). doi:10.1063/1.1692942

4. Max, C., Perkins, F., Strong electromagnetic waves in overdense plasmas. Phys. Rev. Lett. 27, 1342, (1971). doi:10.1103/PhysRevLett.27.1342 